\title{
USE OF A PROTOTYPE AIRBORNE SEPARATION ASSURANCE SYSTEM FOR RESOLVING NEAR-TERM CONFLICTS DURING AUTONOMOUS AIRCRAFT OPERATIONS
}

\author{
Richard Barhydt* \\ NASA Langley Research Center \\ Hampton, VA 23681
}

\author{
Dr. Todd M. Eischeid ${ }^{\dagger}$ \\ Booz-Allen \& Hamilton \\ Hampton, VA 23681
}

\author{
Michael T. Palmer ${ }^{\dagger}$ \\ David J. Wing* \\ NASA Langley Research Center \\ Hampton, VA 23681
}

\begin{abstract}
NASA is currently investigating a new concept of operations for the National Airspace System, designed to improve capacity while maintaining or improving current levels of safety. This concept, known as Distributed Air/ Ground Traffic Management (DAGTM), allows appropriately equipped "autonomous" aircraft to maneuver freely for flight optimization while resolving conflicts with other traffic and staying out of special use airspace and hazardous weather. In order to perform these tasks, pilots use prototype conflict detection, prevention, and resolution tools, collectively known as an Airborne Separation Assurance System (ASAS). While ASAS would normally allow pilots to resolve conflicts before they become hazardous, evaluation of system performance in sudden, near-term conflicts is needed in order to determine concept feasibility.
\end{abstract}

An experiment was conducted in NASA Langley's Air Traffic Operations Lab to evaluate the prototype ASAS for enabling pilots to resolve near-term conflicts and examine possible operational effects associated with the use of lower separation minimums. Sixteen commercial airline pilots flew a total of 32 traffic scenarios that required them to use prototype ASAS tools to resolve close range "pop-up" conflicts. Required separation standards were set at either 3 or 5 NM lateral spacing, with $1000 \mathrm{ft}$ vertical separation being used for both cases. Reducing the lateral separation from 5 to $3 \mathrm{NM}$ did not appear to increase operational risk, as indicated by the proximity to the intruder aircraft. Pilots performed better when they followed tactical guidance cues provided by ASAS than when they didn't follow the guidance. In an effort to improve compliance rate, ASAS design changes are currently under consideration. Further studies will evaluate these design changes and consider integration issues between ASAS and existing Airborne Collision Avoidance Systems (ACAS).

\section{INTRODUCTION}

NASA is investigating a new concept of operations for the future National Airspace System (NAS). This concept, known as Distributed Air/Ground Traffic Management (DAG-TM), enables pilots flying appropriately equipped "autonomous" aircraft to have more flexibility in choosing optimal flight trajectories while simultaneously resolving conflicts with other aircraft, special use airspace, and hazardous weather. ${ }^{1}$ Air Traffic Service Providers still issue traffic flow management constraints to autonomous aircraft and provide traffic separation services to those aircraft unable to participate in autonomous operations. A critical component to the feasibility of DAG-TM operations is the effectiveness of an Airborne Separation Assurance System (ASAS) aboard the autonomous aircraft.

Separation assurance systems would normally enable pilots operating in a DAG-TM environment to respond to traffic conflict situations well before the conflicting aircraft poses a hazard to safe flight. Previous studies have shown that pilots are able to use prototype ASAS tools effectively to resolve longer-term conflicts. ${ }^{2-3}$

One can envision situations that may arise, however, that do not allow this normal opportunity for strategic decision-making. Various non-normal events could require the autonomous aircraft pilot to use the ASAS to resolve a near-term conflict. Examples may include an aircraft descending rapidly due to an emergency or an improper maneuver by an aircraft responding to another conflict. Demonstration of a pilot's ability to effectively use ASAS to resolve near-term conflicts and regain lost

\footnotetext{
*Aerospace Engineer

† Senior Consultant

\$ Senior Member, AIAA, Aerospace Engineer
} 
separation is needed in order to assess concept feasibility.

These types of non-normal events will require the ASAS to provide effective alerts and resolutions prior to the time that an Airborne Collision Avoidance System (ACAS) would give a Resolution Advisory (RA). When an RA is issued, a pilot must take immediate action in order to avoid a potential near miss or collision. The Traffic Alert and Collision Avoidance System (TCAS) II currently functions as an ACAS aboard commercial aircraft. Depending on the own aircraft's altitude, RA's are only issued 15-35 seconds prior to the Closest Point of Approach (CPA). ${ }^{4}$ Prior to an RA, DAG-TM pilots operating autonomous aircraft must rely solely on ASAS for resolution guidance.

An additional area of DAG-TM concept feasibility relates to a potential reduction in separation standards. Lower separation standards will likely improve NAS efficiency and capacity. $^{5-6}$ Current separation minimums are based in large part on the capabilities of older radar systems. ${ }^{7}$ Safety assessments are needed to determine the feasibility of reduced separation minimums. They will give strong consideration to surveillance system performance, including accuracy, integrity, and availability. ${ }^{8-10}$ Candidate surveillance systems include Automatic Dependent SurveillanceBroadcast (ADS-B) and multi-lateration systems. Considering studies done for Reduced Vertical Separation Minimums (RVSM) operations, it is likely that flight technical errors will also be considered. ${ }^{11-12}$

In addition to a thorough evaluation of surveillance system performance, a potential decision to lower the separation standards should also take operational considerations into account. An ASAS Safety Assessment study identified improper maneuvering in response to a conflict (due to ambiguous or improper resolution commands or a pilot's failure to comply with the resolution) as a potential safety risk. ${ }^{9}$ If near-term conflicts with lower separation minimums were determined to be more challenging for pilots, the severity of these risks could be even greater.

An experiment was conducted in NASA Langley's Air Traffic Operations Lab to address issues related to ASAS use for near-term conflicts with a potential reduction in separation minimums. The experiment had the following two primary objectives:

- Evaluate the effectiveness of prototype ASAS tools in enabling the pilot to safely resolve near-term conflicts.

- Compare the effect of 3 and 5 NM lateral separation standards (with $1000 \mathrm{ft}$ vertical separation) on a pilot's ability to safely resolve near-term traffic conflicts.

\section{EXPERIMENTAL APPROACH}

\section{$\underline{\text { Air Traffic Operations Lab }}$}

The Air Traffic Operations Lab at NASA Langley Research Center is a medium fidelity PC workstationbased facility, enabling simultaneous operation by up to 8 subject pilots. Each pilot station consists of a transport aircraft model and flight deck displays designed to replicate the MD-11. Figure 1 shows the display and flight control suite made available to the pilots during the experiment. Traffic information was superimposed on the navigation display and pilots were provided with a Navigation Display Control Panel (NDCP) to adjust the display's traffic-specific features. The NDCP was located to the left of the Glareshield Control Panel. All control of the simulation aircraft was done through the Glareshield Control Panel and Multifunction Control Display Unit (MCDU) associated with the Flight Management System (FMS). No manual flight control was available. 


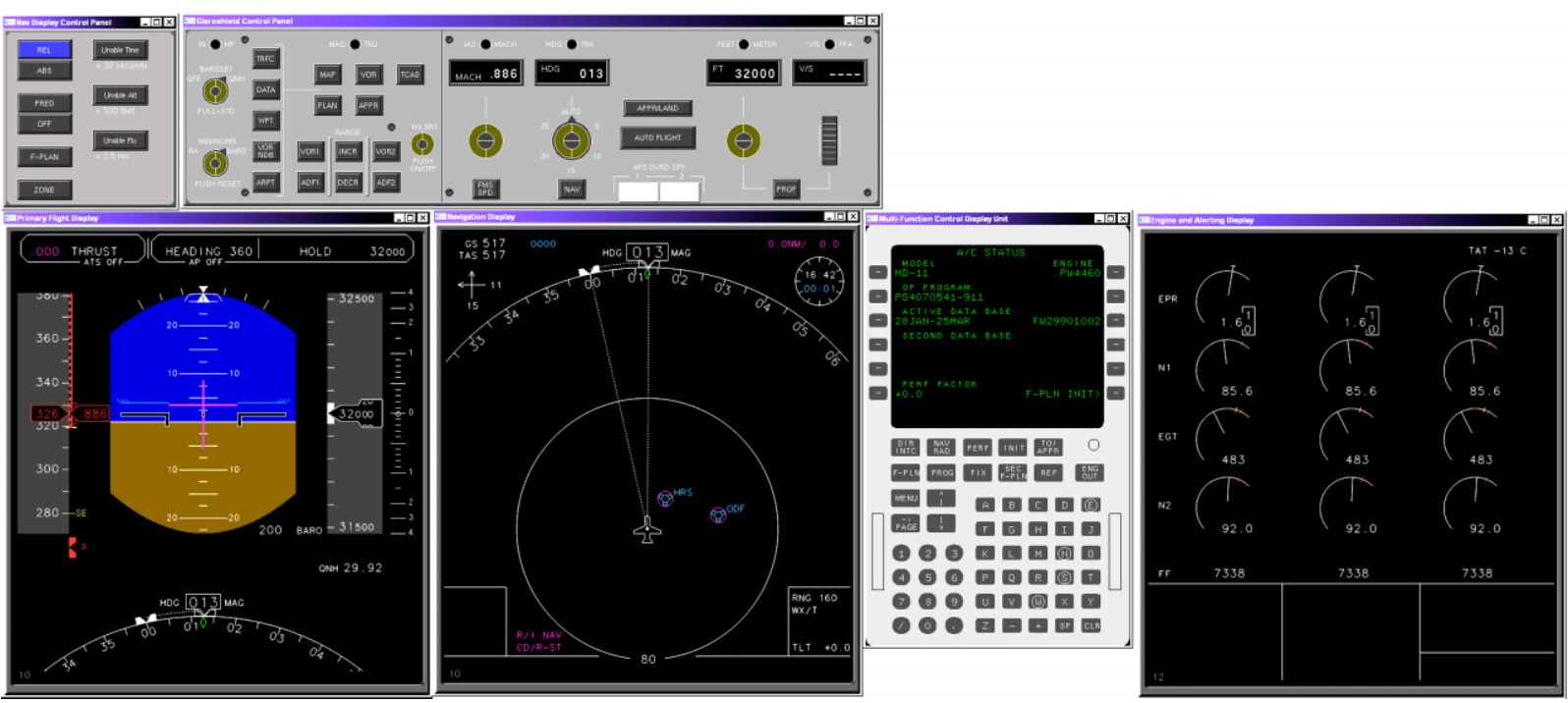

Figure 1. Pilot Displays and Control Panels

$\underline{\text { Subjects }}$

Sixteen commercial airline pilots with experience in Airbus glass cockpit or MD-11 aircraft participated in the experiment. All were active pilots or had retired within the previous year. Their ages ranged from 32 to 57 and flight experience ranged from 4,200 to 23,000 hours.

\section{$\underline{\text { Design }}$}

The experiment used a single-factor within-subjects design. This factor was the required lateral separation from other aircraft and was set at either 3 or $5 \mathrm{NM}$. The required vertical separation was $1000 \mathrm{ft}$ for both cases.

Pilots flew one scenario for each lateral separation condition and each scenario had one designed near-term conflict. Although other background aircraft were present, the data analysis only considered the designed conflict. Scenarios for the lateral separation condition were counterbalanced for order and were included as part of a larger study that consisted of nine scenarios flown per pilot. Several of the other seven scenarios were run in between those for this experiment, in order to help prevent the pilot from anticipating the designed conflict. This paper only discusses results from the two near-term conflict scenarios.

\section{$\underline{\text { Scenario Set-up and Pilot Tasks }}$}

All pilots were flying autonomous aircraft in a DAG$\mathrm{TM}$ en-route environment and were asked to maintain standard separation from other air traffic and from restricted airspace. Pilots were allowed to maneuver freely without contacting a controller. This experiment focused only on air-air separation assurance involving "autonomous" aircraft and the simulation did not incorporate a ground component. Future studies are planned that will look at integrated air-ground applications involving aircraft of mixed equipage.

In addition to maintaining traffic separation, pilots were given a downstream waypoint to cross at a Required Time of Arrival (RTA). En route to the RTA, the aircraft's programmed flight path went through a 65 NM wide corridor with restricted airspace areas on each side. Pilots were asked to continue to the RTA waypoint when able after resolving a conflict. The designed conflict occurred about 15 minutes into a 25minute scenario.

In order to simulate an unexpected near-term conflict, the designated intruder aircraft was hidden from the subject pilot until just before the predicted loss of separation. At this point, a "pseudo pilot" would turn the intruder toward the subject and turn on the intruder's ADS-B broadcast. It appeared about $6 \mathrm{NM}$ away from and at co-altitude with ownship. An alert occurred shortly thereafter. 
The initial conflict geometry (proximity, approach angle, and time to closest approach) was designed to be the same for both $3 \mathrm{NM}$ and $5 \mathrm{NM}$ separation zone cases. Pseudo pilots were used to ensure the conflict occurred, even if the subject pilot had deviated from the original programmed flight plan. Use of the same initial conflict conditions for all scenarios allowed a comparison of risk incurred between the $3 \mathrm{NM}$ and $5 \mathrm{NM}$ separation zone conditions.

For the $5 \mathrm{NM}$ separation zone condition, separation loss occurred earlier, but further away from the intruder when compared to a $3 \mathrm{NM}$ zone. These differences would likely highlight any variation in pilot performance due to an aircraft being inside or outside the separation zone. As discussed below, alert symbology changed slightly when separation was lost.

Because the intruder in the conflict scenarios always appeared close to the ownship, a highly aggressive maneuver would have been required to avoid a separation violation. In order to evaluate a pilot's ability to regain separation, avoiding a separation loss was designed to be nearly impossible for the scenarios used in this experiment.

Depending on selected map range, the subject pilot could see additional scripted aircraft on the traffic display while en-route to the RTA waypoint. An automatic traffic filter showed other aircraft determined to be operationally significant, based on proximity, current trajectory, and intent.

After each scenario, pilots were asked to complete a questionnaire asking them to rate the effectiveness of the traffic alerts and resolution guidance in resolving the conflicts. They were also asked to assess the safety risk posed by the pop-up conflicts.

\section{$\underline{\text { Displays and Alerts }}$}

The prototype ASAS combined a state-based (position, ground speed, and ground track) tactical system with a strategic system based on state and FMS intent information. ASAS tools were part of larger decision aiding system, known as the Autonomous Operations Planner. ${ }^{13}$ TCAS was not available for the experiment.

Traffic alerts were based on outer and inner zones surrounding the aircraft, referred to as the separation and collision zones, respectively (see Figure 2). This design was based on the concept described by RTCA's Airborne Conflict Management (ACM) committee. ${ }^{10}$ The separation zone represents the minimum legal separation around an aircraft. It had a 3 or $5 \mathrm{NM}$ radius, depending on the scenario. The vertical dimension was $1000 \mathrm{ft}$ for both cases. A conflict was defined as a predicted loss of separation and a separation violation occurred if an aircraft penetrated another aircraft's separation zone. The collision zone's radius and height above/below were $900 \mathrm{ft}$ and $300 \mathrm{ft}$, respectively. Penetration of the collision zone was considered to be a near miss.

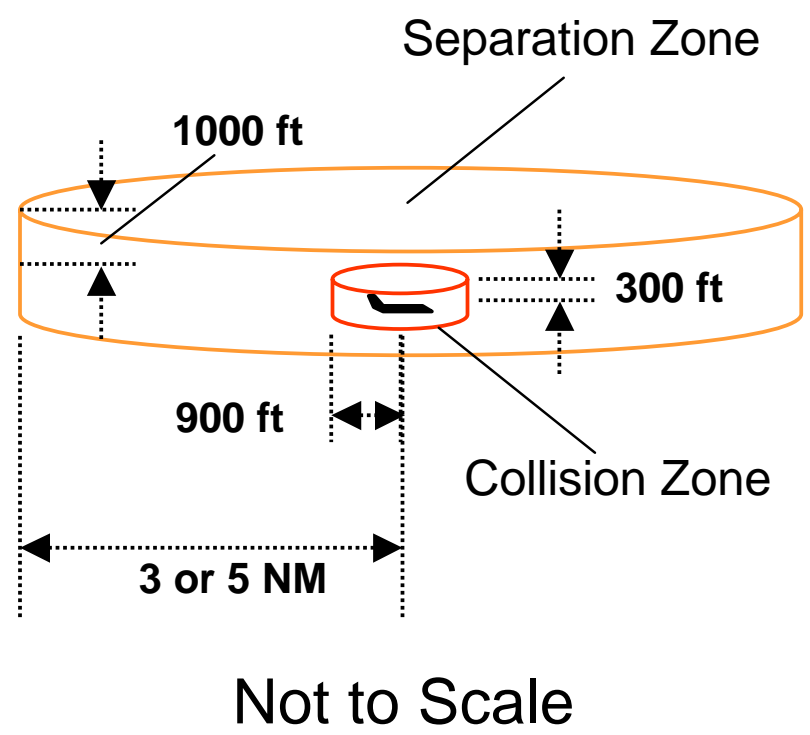

Figure 2. Separation and Collision Zones

Figure 3 shows a notional timeline for ASAS and ACAS as applied to this experiment. After a conflict was detected but prior to separation loss, ASAS provided information to assist the pilot in maintaining adequate separation. Strategic and/or tactical separation assurance decision support tools were available, depending on the time to conflict. The strategic resolution system was integrated with the FMS to provide an efficient conflict resolution and return to the programmed flight plan. Due to the short timeframe of the planned conflicts, pilots were not able to use strategic resolutions for this study. Tactical conflict resolutions used only state information and provided recommended changes in heading and/or vertical speed. Tactical resolutions became available 5 minutes prior to the conflict and strategic resolutions were phased out 2 minutes prior to conflict. This transition region was outside the timeframe of the pop-up traffic. Further details of these systems are provided by Wing et al. ${ }^{3,14}$ 
If separation was lost, tactical resolutions continued to provide guidance to the pilot on ways to regain the minimum separation. In these cases, pilots were instructed in a pre-flight briefing to follow the tactical guidance. If applicable, an alert was issued one minute prior to predicted collision zone entry (considered on the timeline to be close to the CPA). Note that the loss of separation could shift left or right and occur before or after a collision zone alert, depending on the relative velocity between the two aircraft. For this experiment, a collision zone alert, if issued, normally occurred prior to separation loss. Issuance of this alert did not affect the tactical resolutions.
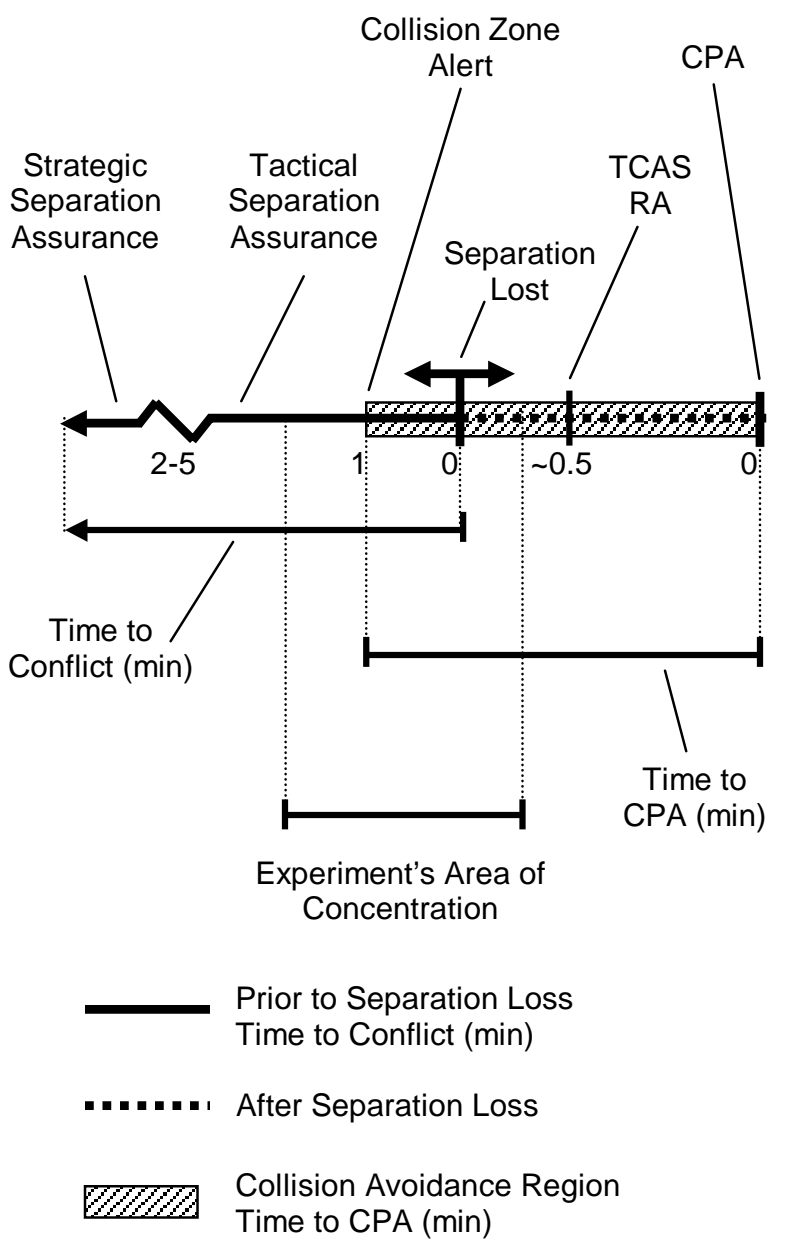

Notes: Times Not to Scale TCAS Not Available for Experiment

Figure 3. Notional ASAS/ACAS Timeline
The experiment focused on maneuvers performed by pilots just prior to when TCAS would have provided an RA. As discussed earlier, TCAS issues RA's between 15 and 35 seconds prior to CPA. Conflict alerts during the experiment occurred mainly between 60-70 seconds prior to the CPA. This timeframe may be considered to be near a transition between separation assurance and collision avoidance. Because TCAS was not incorporated, no explicit effort was made to consider integration of ACAS RA's and ASAS resolutions in this simulation.

Figure 4 shows Primary Flight and Navigation/Traffic Displays as typically seen by the pilot shortly after the intruder aircraft appeared. The intruder was well inside the conflict detection threshold and therefore the conflict alert was generated almost immediately. This alert was shown as an amber band along the ownship's flight path where the separation loss was predicted to occur. The intruder was also color-coded amber to represent its threat level.

When a state-based conflict was detected, pilots were presented with a tactical resolution, shown as green bugs on the vertical speed and heading indicators. The bugs showed the recommended change in either heading or vertical speed needed to resolve the conflict. This resolution guidance allowed the pilot to resolve the conflict by following either the lateral or vertical guidance alone. The resolution algorithm used a modified voltage potential method, originally developed by Eby ${ }^{15}$ and refined by the NLR ${ }^{16}$. This algorithm acts to increase the projected separation between the two aircraft at CPA.

Conflict prevention bands were placed on the heading and vertical speed indicators to show headings and vertical speeds that, if flown, would cause a conflict with another aircraft. These bands were predicted using current state information for up to a five-minute time horizon. The heading and vertical speed bands assumed a constant ground speed maneuver in either the horizontal or vertical plane, respectively. A change in speed or a combined lateral/vertical maneuver would change the conflict prevention bands. Once inside another aircraft's separation zone, conflict prevention bands were removed. After separation loss, the bands no longer provided useful information because they would span the entire heading and vertical speed range. 


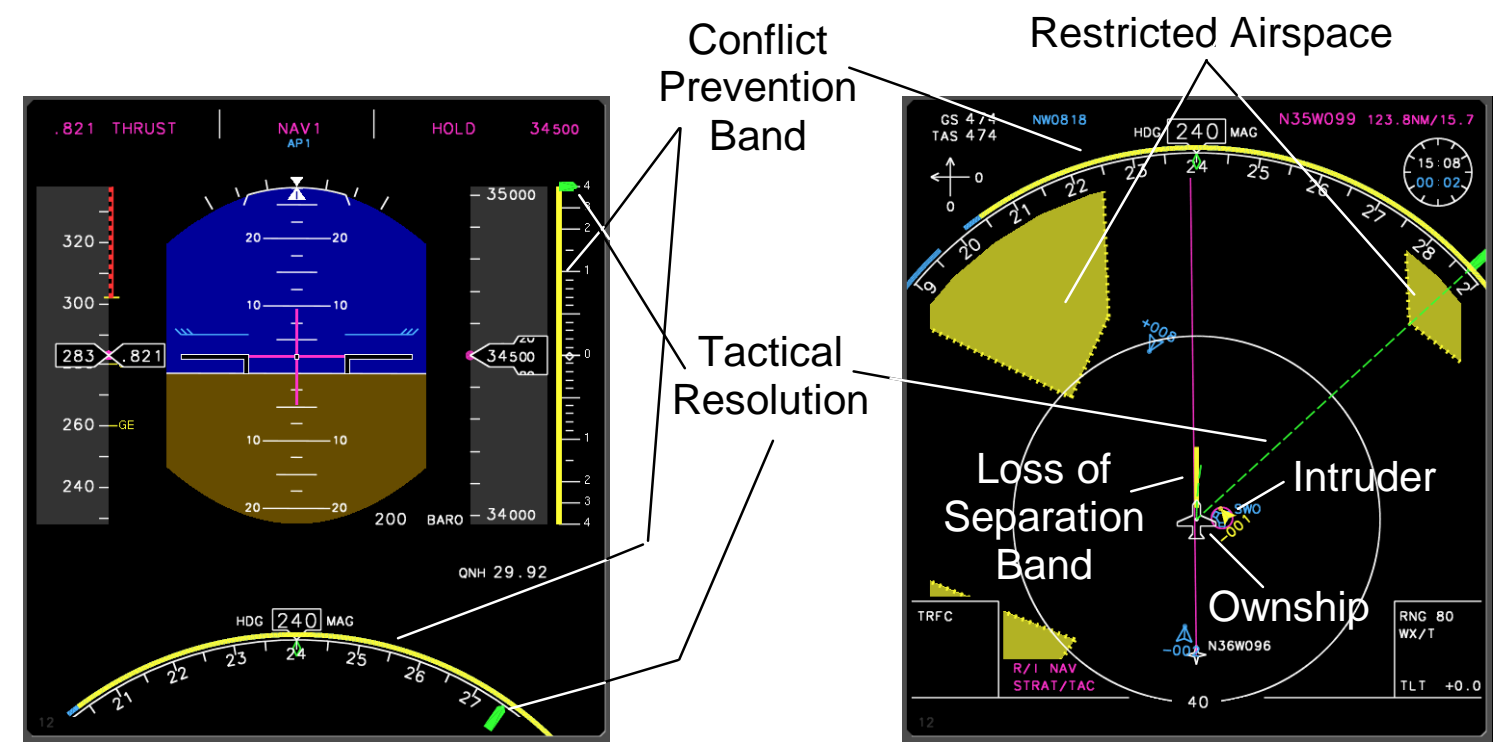

Figure 4. Primary Flight (left) and Navigation (right) Displays

\section{Results}

\section{$\underline{\text { Performance Metrics }}$}

A single parameter that combines the relative lateral and vertical distances between the ownship and intruder aircraft was used as a measure of threat severity. This parameter, referred to as epsilon ( $\varepsilon$ ), is discussed by den Braven ${ }^{17}$. It represents the ellipse distance of an ellipsoid enclosed within the cylindrical separation zone.

Figure 5 shows a cross-section of this geometry for an ellipsoid corresponding to a $5 \mathrm{NM}$ separation standard. The lengths of the ellipsoid's major and minor axes are the separation zone's diameter and total height, respectively. When $\varepsilon=1$, an aircraft is on the surface of the ellipsoid centered on another aircraft. Once inside the separation zone, threat severity increases as $\varepsilon$ approaches zero.

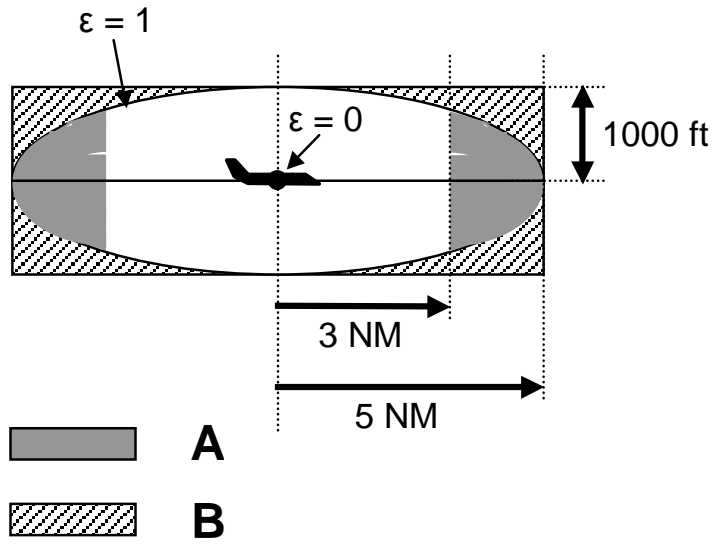

Figure 5. Ellipsoid and Cylindrical Separation Zone
In order to compare results from the 3 and $5 \mathrm{NM}$ scenarios, all results using $\varepsilon$ were calculated with a 5 NM separation zone. With this convention, the same value of $\varepsilon$ corresponded to the same threat level for both separation standards. For cases where the tested separation zone was $3 \mathrm{NM}, \varepsilon<1$ did not necessarily imply a separation violation (Figure 5, Region A). Conversely, a separation violation may still have occurred when $\varepsilon>1$, for cases where the aircraft was outside the ellipsoid, but within the separation zone cylinder (Figure 5, Region B).

Two performance metrics were considered for the experiment:

- Threat Proximity $\left(\varepsilon_{\min }\right)$ : actual minimum $\varepsilon$ between the two aircraft.

- Risk Mitigation $\left(\varepsilon_{\text {diff }}\right)$ difference between the predicted minimum $\varepsilon$ at the time the alert was issued (based on current state information for both aircraft) and $\varepsilon_{\text {min. }}$

\section{Threat Proximity}

Figure 6 shows $\varepsilon_{\min }$ for the 3 and 5 NM separation cases, broken down by whether the pilot complied or did not comply with the tactical resolution. The pilot was said to comply if his initial maneuver (either lateral or vertical) was in the same direction as the corresponding tactical resolution. Using this definition, the pilot complied $9 / 15(60 \%)$ times for 
the 3 NM separation zone and 13/16 (81\%) times for the 5 $\mathrm{NM}$ zone. Note that one $3 \mathrm{NM}$ separation scenario was lost due to an earlier pilot maneuver that prevented the planned conflict from taking place.

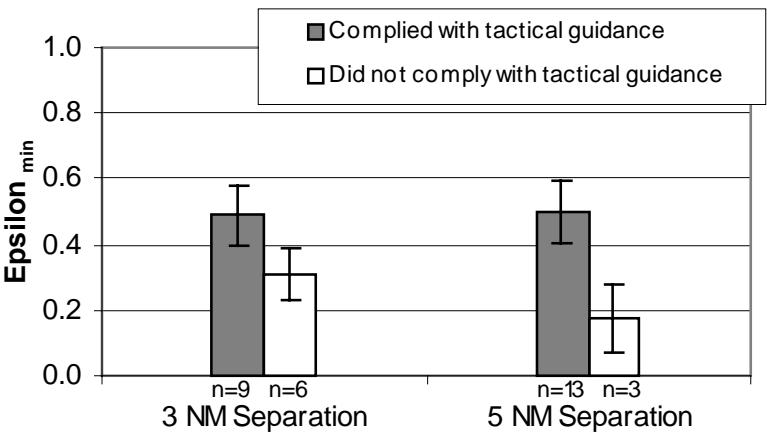

Note: Error bars represent 1 standard error of the mean.

Figure 6. Minimum Epsilon by Separation Zone Size and Tactical Resolution Compliance

The lateral separation zone distance did not appear to affect threat proximity. No significant differences were found for $\varepsilon_{\min }$ between the 3 and $5 \mathrm{NM}$ separation cases when combined over pilot compliance.

A linear regression of tactical guidance compliance (combined across separation zone conditions) onto $\varepsilon_{\min }$ was performed. The regression showed that compliance could significantly predict $\varepsilon_{\min }(F(1,29)=4.264, p<.05$, $\left.R_{\text {adj }}^{2}=.098\right)$ and that $\varepsilon_{\text {min }}$ was larger for those who complied $(M=.497, S D=.310, n=22)$ than for those who did not comply $(M=.266, S D=.193, n=9)$. Observed power $(1-\beta)$ was .515 .

\section{$\underline{\text { Risk Mitigation Due to Maneuver }}$}

Whereas $\varepsilon_{\min }$ is a measure of maximum threat severity, $\varepsilon_{\text {diff }}$ can be used to assess a pilot's ability to improve the conflict situation over that predicted when the alert was first issued. Epsilon $_{\text {diff }}$ is a more suitable metric for comparing pilot performance because it accounts for any differences in predicted $\varepsilon_{\min }$ that may have occurred due to different initial conflict geometry. Although the experiment was designed to generate the same initial conditions (proximity, approach angle and time to closest point of approach) across all scenarios, prior maneuvers by the subject pilot caused differences in a few cases. On average, however, these differences were minimal. Predicted $\varepsilon_{\min }$ at the time of the alert ranged from 0.01 for the $3 \mathrm{NM}$ /did-not-comply scenarios to 0.07 for the 5
NM/comply cases, with an overall mean across all scenarios of 0.04 .

Figure 7 shows $\varepsilon_{\text {diff }}$ as a function of separation zone size and resolution compliance. A linear regression of tactical guidance compliance (combined across separation zone conditions) onto $\varepsilon_{\text {diff }}$ showed that compliance could marginally predict $\varepsilon_{\text {diff }}(F(1,29)=$ $\left.3.118, p=0.088, R_{\text {adj }}^{2}=.066\right)$ and that $\varepsilon_{\text {diff }}$ was marginally larger for those who complied with guidance $(M=.446, S D=.297, n=22)$ than those who did not comply $(M=.256, S D=.192, n=9)$. Observed power $(1-\beta)$ was .400 . This result was not significant at the $\alpha=.05$ level used for the present study.

The lateral separation zone distance did not appear to affect the pilot's risk mitigation. No significant differences were found for $\varepsilon_{\text {diff }}$ between the 3 and 5 NM separation cases when combined over pilot compliance.

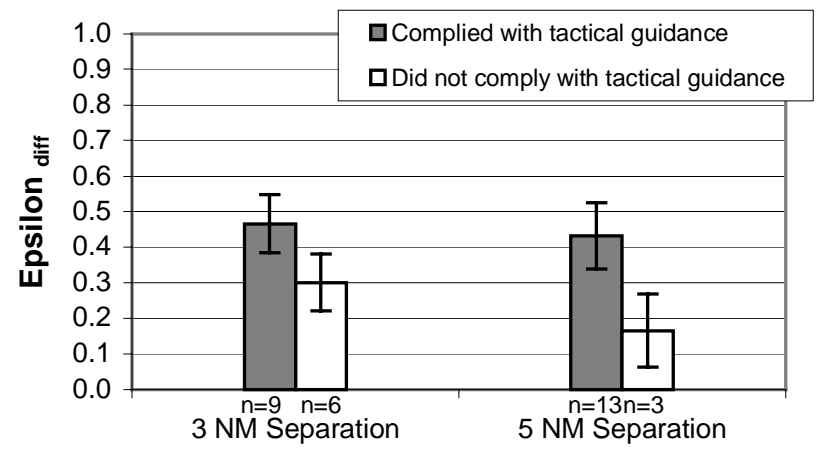

Note: Error bars represent 1 standard error of the mean.

Figure 7. Differences between Actual Minimum Epsilon and Minimum Epsilon Predicted when Alert Issued, by Separation Zone Size and Tactical Resolution Compliance

\section{$\underline{\text { Pilot Questionnaires }}$}

Post-scenario questionnaire results related to the decision support tools and the pilots' perceived operational safety are shown in Table 1. Each question had a 1 to 7 rating scale, ranging from 1 (least favorable) to 7 (most favorable). The scale description as applied to each question is given in the $2^{\text {nd }}$ column of Table 1. 
Table 1. Pilot Questionnaire Results

\begin{tabular}{|l|l|l|}
\hline Question & Rating Scale & $\begin{array}{l}\text { Overall } \\
\text { Mean }\end{array}$ \\
\hline $\begin{array}{l}\text { How intuitive } \\
\text { was the conflict } \\
\text { alerting system? }\end{array}$ & $\begin{array}{l}1: \text { not at all } \\
\text { intuitive } \rightarrow \text { 7: } \\
\text { very intuitive }\end{array}$ & 5.0 \\
\hline $\begin{array}{l}\text { How acceptable } \\
\text { were the tactical } \\
\text { resolutions? }\end{array}$ & $\begin{array}{l}1: \text { acceptable at all } \\
\text { completely } \\
\text { acceptable }\end{array}$ & 4.3 \\
\hline $\begin{array}{l}\text { What was the } \\
\text { level of safety for } \\
\text { this scenario? }\end{array}$ & $\begin{array}{l}1: \text { completely } \\
\text { completely safe }\end{array}$ & 4.0 \\
\hline $\begin{array}{l}\text { How did the } \\
\text { conflict manage- } \\
\text { ment tools affect } \\
\text { the risk level? }\end{array}$ & $\begin{array}{l}1: \text { greatly } \\
\text { increased risk } \rightarrow \\
7: \text { greatly } \\
\text { decreased risk }\end{array}$ & 4.8 \\
\hline
\end{tabular}

Results suggest that pilots had a weak acceptance of the conflict alerts and resolutions. These results may be partially due to pilots only having an opportunity to use the ASAS tools during high-risk short-term conflicts. Potential design changes to the ASAS tools currently under consideration and discussed below may also improve these ratings for future studies. It is not surprising that pilots felt neutral about the level of safety corresponding to a pop-up conflict. These conflicts were designed to pose a safety hazard associated with a nonnormal event.

The DAG-TM concept incorporates several safety measures to reduce the likelihood of the type of scenario evaluated in this experiment ${ }^{1}$. Before maneuvering, pilots of autonomous aircraft must ensure that they do not create near-term conflicts. Refined ASAS tools relying on highly dependable surveillance systems should provide these aircraft with adequate warning of upcoming conflicts in all nominal cases. During normal operations, pilots of both aircraft will have the necessary tools to resolve conflicts. New decision support tools are also expected to further assist air traffic service providers in providing separation services for aircraft not equipped for autonomous operations.

\section{Discussion and ASAS Enhancements}

Reducing the lateral separation from 5 to $3 \mathrm{NM}$ did not appear to increase operational risk for the near-term conflicts studied in this experiment. Additional studies could consider whether any reduction in alert and subsequent maneuver times would lead to lower risk in cases where a conflict situation exists prior to the alerting time horizon. Because a 3 NM separation zone represents a smaller target, trajectory uncertainties may cause a shorter alert time when compared to a $5 \mathrm{NM}$ zone. A smaller separation zone may also reduce the number of alerted conflicts that evolve into safety critical situations. Due to the consistent alert time associated with the pop-up conflicts in this experiment, no such changes could be observed. As discussed earlier, extensive analysis is also needed to determine whether surveillance system performance can support lower separation minimums.

Pilots were able to reduce threat severity and mitigate risk in response to pop-up conflicts more effectively when they followed the tactical guidance from ASAS. In order to further improve upon these results, several design enhancements are currently under consideration for the conflict detection, prevention, and resolution systems. These changes focus on a more integrated approach for showing information to pilots. Previous research suggests that when provided with alerts that are consistent with displayed information, pilots are more likely to comply promptly with resolution guidance. ${ }^{18}$

As discussed above, the prototype ASAS employed during this experiment consisted of tactical and strategic separation assurance. Tactical information included state-based conflict prevention and tactical resolutions. The strategic system detected conflicts using a combination of state and FMS-based intent information and provided FMS resolutions. These logic differences may have caused the tactical and strategic systems to display different alert levels for a threat aircraft.

Although pilots did not observe the transition from strategic to tactical separation assurance for the shortterm conflicts studied in this experiment, they did fly several scenarios with long-term conflicts as part of a larger study. ${ }^{19-20}$ If pilots encountered cases of nonsupporting (dissonant) alerts between the strategic and tactical systems, their overall confidence in the ASAS may have been adversely affected. ${ }^{21}$

Initial design enhancements to the prototype ASAS have focused on the interaction between the state- 
based Conflict Prevention (CP) system and the Conflict Detection (CD) system that considers both state and FMS intent. The latter produces a Loss of Separation (LoS) band along the ownship flight path where a separation violation is predicted to occur. Knowledge of intent information can cause the LoS band to reflect a different level of alert than the state-based CP band on the map display's compass rose. The $\mathrm{CP}$ band shows a range of headings that, if flown, would cause a conflict with another aircraft. Incorporating FMS intent information into the $\mathrm{CP}$ system will enable the $\mathrm{CD}$ and $\mathrm{CP}$ systems to generate consistent alerts, thereby enabling pilots to achieve a more unified mental model of conflict situations.

The left pane of Figure 8 shows a "false negative" situation where the existing $\mathrm{CP}$ system does not validate a detected intent-based conflict. The LoS band indicates that a traffic aircraft is in conflict with ownship, however, no band is visible on the heading arc. Since a conflict exists, a CP band should encompass the present heading, as shown in the redesigned display (right pane of Figure 8).
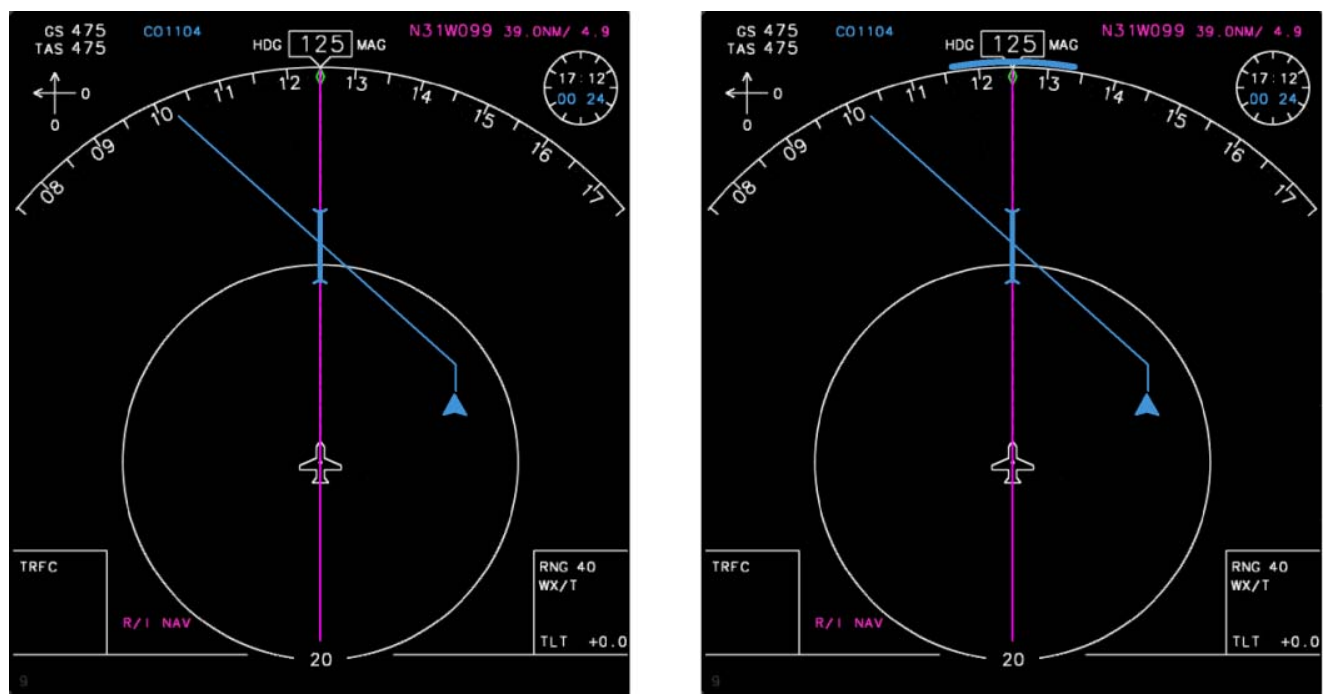

Figure 8. "False Negative" Situation Occurring with Current Display (left) and Re-designed Display Incorporating Intent-based CP Logic (right).

Conversely, the "false positive" is another situation that may cause a difference in conflict indications. The left pane of Figure 9 illustrates how such a scenario could develop with the existing display. The traffic aircraft on the right has a flight path that does not place it in conflict with ownship. Using the state projection, the $\mathrm{CP}$ system believes that a conflict does exist and draws a band over the current heading. This indication may suggest a maneuver when none is required. In contrast, the CD system has knowledge of intent information and does not detect a conflict. The enhanced CP system shown in the right pane of Figure 9 considers traffic aircraft intent information. 

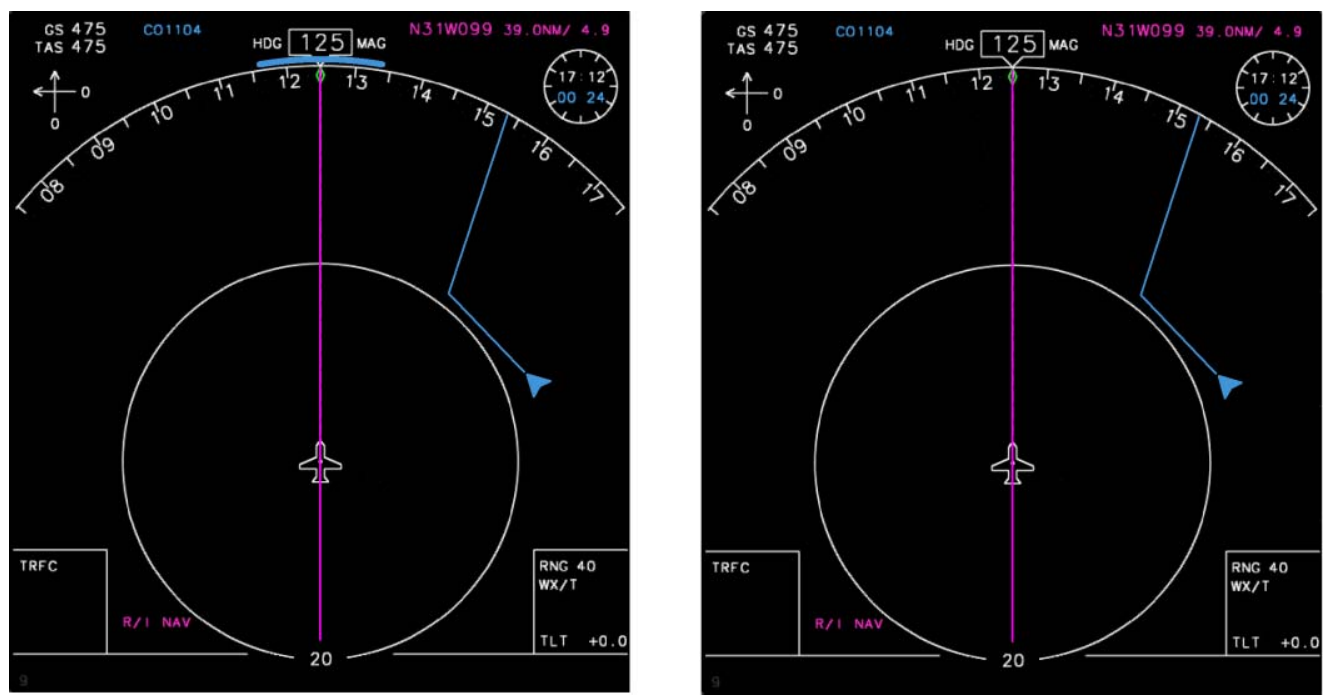

Figure 9. "False Positive" Situation Occurring with Current Display (left) and Re-designed Display Incorporating Intent-based CP Logic (right).

In addition to the integration of $\mathrm{CD}$ and $\mathrm{CP}$ systems, design enhancements to the tactical resolution system are also under consideration. These changes could tap into the extensive development and evaluation of TCAS. As the time to closest point of approach decreases, it becomes more important for the ASAS resolutions to be compatible with RA's that are eventually provided by ACAS. ${ }^{8}$ TCAS design goals that could be considered for ASAS enhancement include: attempt to avoid crossing the intruder's altitude (especially for very close encounters), provide a resolution that does not require the ownship to change direction if currently maneuvering, allow time for each aircraft to initiate a maneuver prior to reversing the direction of an RA, and only reverse an RA if needed to ensure safety., ${ }^{422-23}$ These design goals are likely extensible to both lateral and vertical maneuvers. For example, previous studies have shown that pilots are less likely to turn toward the intruder as they get closer to the conflict. ${ }^{24}$

\section{Conclusions}

For near-term conflicts, a pilot's ability to reduce threat proximity and mitigate risk appears to depend more on compliance with the ASAS resolution guidance than on the size of the lateral separation zone. Compliance with the tactical resolutions led to improvement in threat proximity and risk mitigation. These positive effects may be further increased by design enhancements to the prototype ASAS.

Before air-air separation assurance or other applications with lower separation minimums are implemented, a thorough Operational Safety Assessment will need to be performed. This process has been described by Zeitlin. ${ }^{8}$ Included in this process is an Operational Hazard Analysis where potential hazards, the operational effects of those hazards, and mitigation strategies are assessed. Evaluation of pilot performance using ASAS tools will likely be an important part of this process.

\section{References}

${ }^{1}$ NASA, "Concept Definition for Distributed Air/Ground Traffic Management (DAG-TM)," Version 1.0., 1999.

${ }^{2}$ Cashion, P. and Lozito, S., "The Effects of Different Levels of Intent Information on Pilot Self Separation Performance," Proceedings of the 10th International Symposium on Aviation Psychology, Vol. 1, Ohio State University, Columbus, OH, 1999, pp. 177-182.

${ }^{3}$ Wing, D., Barmore, B., and Krishnamurthy, K., "Airborne Use of Traffic Intent Information in a Distributed Air-Ground Traffic Management Concept: 
Experiment Design and Preliminary Results," 4th USA/Europe ATM R\&D Seminar, Santa Fe, NM, 2001.

${ }^{4}$ FAA, "Introduction to TCAS II Version 7," www.arinc.com/downloads/tcas/tcas.pdf, 2000.

${ }^{5}$ RTCA, "Final Report of the RTCA Task Force 3: Free Flight Implementation,” Washington DC, Oct. 1995.

${ }^{6}$ Casaux, F. and Hasquenoph, B., "Operational Use of ASAS," 1st USA/Europe ATM R\&D Seminar, Saclay, France, 1997.

${ }^{7}$ Reynolds, T. and Hansman, R.J., "Analysis of Separation Minima Using a Surveillance State Vector Approach," 3rd USA/Europe ATM R\&D Seminar, Napoli, Italy, 2000.

${ }^{8}$ Zeitlin, A. and Bonnemaison, B., "Managing Criticality of ASAS Applications," 3rd USA/Europe ATM R\&D Seminar, Napoli, Italy, 2000.

${ }^{9}$ Zeitlin, A., "Safety Assessments of ADS-B and ASAS," 4th USA/Europe ATM R\&D Seminar, Santa Fe, NM, 2001.

${ }^{10}$ RTCA, "Application of Airborne Conflict Management," RTCA/DO-263, Washington, DC, 2000.

${ }^{11}$ Joyce, K., "Developing Monitoring Requirements for Reduced Separation Airspace," Proceedings of the Aerospace Technology Conference and Exposition, SAE, Long Beach, CA, 1990.

${ }^{12}$ Harrison, D. and Moek, G., "European Studies to Investigate the Feasibility of Using $1000 \mathrm{ft}$ Vertical Separation Minima above FL 290. Part II Precision Radar Data Analysis and Collision Risk Assessment," Journal of Navigation, Vol. 45, 1992, pp. 91-106.

${ }^{3}$ Ballin, M. et al., "A Flight Deck Decision Support Tool for Autonomous Airborne Operations," AIAA Paper 2002-4554, Aug. 2002.

${ }^{14}$ Wing, D., B. Barmore, and K. Krishnamurthy, "Use of Traffic Intent Information by Autonomous Aircraft in Constrained Operations," AIAA Paper 2002-4555, Aug. 2002.

${ }^{15}$ Eby, M., "A Self-Organizational Approach for Resolving Air Traffic Conflicts," The Lincoln
Laboratory Journal, Vol. 7, No. 2, MIT, 1994, pp. 239254.

${ }^{16}$ Hoekstra, J. et al., "Overview of NLR Free Flight Project 1997-1999," Contract Report NLR-CR-2000227, National Aerospace Laboratory, 2000.

${ }^{17}$ den Braven, W., "Analysis of Aircraft/Air Traffic Control System Performance,” AIAA Paper 95-3363, Aug. 1995.

${ }^{18}$ Pritchett, A., "Pilot Situation Awareness and Alerting System Commands," Proceedings of the Advances in Aviation Safety Conference and Exposition, SAE, Daytona Beach, FL, 1998, pp. 145-155.

${ }^{19}$ Barmore, B., Johnson, E., Wing, D.J., and Barhydt, R., "Airborne Conflict Management within Confined Airspace in a Piloted Simulation of DAG-TM Autonomous Aircraft Operations," 5th USA/Europe ATM R\&D Seminar, Budapest, Hungary, 2003.

${ }^{20}$ Wing, D.J., Krishnamurthy, K., Barhydt, R., and Barmore, B., "Pilot Interactions in an Over-constrained Conflict Scenario as Studied in a Piloted Simulation of Autonomous Aircraft Operations," 5th USA/Europe ATM R\&D Seminar, Budapest, Hungary, 2003.

${ }^{21}$ Song, L. and Kuchar K., "Describing, Predicting, and Mitigating Dissonance between Alerting Systems," $4^{\text {th }}$ International Workshop on Human Error, Safety, and System Development, Linkoping, Sweden, 2001.

${ }^{22}$ Love, W.D., "TCAS III: Bringing Operational Compatibility to Airborne Collision Avoidance," AIAA Paper 88-4004, Oct. 1988.

${ }^{23}$ Chappell, S., "Avoiding a Maneuvering Aircraft with TCAS," Proceedings of the 5th International Symposium on Aviation Psychology, Vol. 1, Ohio State University, Columbus, OH, 1989, pp. 338-343.

${ }^{24}$ Smith, J., Ellis, S., and Lee, E., "Perceived Threat and Avoidance Maneuvers in Response to Cockpit Traffic Displays," Human Factors Journal, Vol. 26, Human Factors Society, Feb. 1984, pp. 33-48. 